\title{
Criminologie
}

\section{L'évaluation des signalements à la Direction de la protection de la jeunesse : étude des facteurs qui influencent les décisions prises par les intervenants}

\section{Marie Jacob et Danielle Laberge}

Volume 34, numéro 1, printemps 2001

La notion de risque dans la gestion pénale

URI : https://id.erudit.org/iderudit/004753ar

DOI : https://doi.org/10.7202/004753ar

Aller au sommaire du numéro

Éditeur(s)

Les Presses de l'Université de Montréal

ISSN

0316-0041 (imprimé)

1492-1367 (numérique)

Découvrir la revue

Citer cet article

Jacob, M. \& Laberge, D. (2001). L'évaluation des signalements à la Direction de la protection de la jeunesse : étude des facteurs qui influencent les décisions prises par les intervenants. Criminologie, 34(1), 123-155.

https://doi.org/10.7202/004753ar
Résumé de l'article

L'objectif de cette recherche est d'identifier les facteurs qui influencent la prise de décision dans les services de protection de la jeunesse. Deux décisions sont plus spécifiquement examinées : 1) la décision de retenir le signalement et 2) la décision visant à déterminer si la sécurité ou le développement de l'enfant est compromis. L'étude porte sur un échantillon représentatif de signalements $(n=720)$ reçus au Centre jeunesse de Québec en 1994 et 1995. Les données ont été recueillies à partir d'un questionnaire d'enquête rempli par les intervenants et des données consignées dans les dossiers des enfants. Dans l'ensemble, la sécurité et le développement de l'enfant sont considérés comme compromis dans $17,2 \%$ des cas, c'est-à-dire pour un pourcentage relativement faible d'enfants signalés. Selon les résultats des analyses de régression logistique effectuées, la source du signalement, le type de problème signalé, la personne mise en cause, l'unité administrative ayant reçu le signalement et le statut de l'enfant dans l'établissement sont les facteurs qui permettent le mieux de prédire l'issue des décisions rendues. On observe à la fois des similitudes et des différences entre les deux décisions étudiées. Les résultats de la recherche soulèvent de nombreux enjeux liés à l'évaluation des signalements et à l'orientation des enfants signalés. 


\title{
L'évaluation des signalements à la Direction de la protection de la jeunesse : étude des facteurs qui influencent les décisions prises par les intervenants ${ }^{*}$
}

\author{
Marie Jacob \\ Centre de recherche sur les \\ services communautaires \\ Université Laval \\ marie.jacob@crsc.ulaval.ca \\ Danielle Laberge \\ Professeur \\ Département de sociologie \\ Université du Québec à Montréal \\ laberge.danielle@uquam.ca
}

RÉSUMÉ - L'objectif de cette recherche est d'identifier les facteurs qui influencent la prise de décision dans les services de protection de la jeunesse. Deux décisions sont plus spécifiquement examinées : 1 ) la décision de retenir le signalement et 2) la décision visant à déterminer si la sécurité ou le développement de l'enfant est compromis. L'étude porte sur un échantillon représentatif de signalements $(n=720)$ reçus au Centre jeunesse de Québec en 1994 et 1995. Les données ont été recueillies à partir d'un questionnaire d'enquête rempli par les intervenants et des données consignées dans les dossiers des enfants. Dans l'ensemble, la sécurité et le développement de l'enfant sont considérés comme compromis dans $17,2 \%$ des cas, c'est-à-dire pour un pourcentage relativement faible d'enfants signalés. Selon les résultats des analyses de régression

* Cette étude a bénéficié de financements du Conseil de recherche en sciences humaines du Canada et du Fonds pour la Formation de Chercheurs et l'Aide à la Recherche Les auteures désirent remercier le personnel du Comité de protection de la jeunesse de Québec pour sa collaboration constante dans la poursuite de ce projet. Les auteures désirent aussi remercier Louis-Paul Rivest et Gaétan Daigle du Départment de mathématiques et de statistiques et Marie Simard de l'École de service social de l'Université Laval pour leurs commentaires et leurs suggestions.

Criminologie, vol. 34, $n^{\circ} 1$ (2001) 
logistique effectuées, la source du signalement, le type de problème signalé, la personne mise en cause, l'unité administrative ayant reçu le signalement et le statut de l'enfant dans l'établissement sont les facteurs qui permettent le mieux de prédire l'issue des décisions rendues. On observe à la fois des similitudes et des différences entre les deux décisions étudiées. Les résultats de la recherche soulèvent de nombreux enjeux liés à l'évaluation des signalements et à l'orientation des enfants signalés.

ABSTRACT - The purpose of this study is to investigate the determining factors in the decision-making process in child protective services. Two specific issues are examined: 1 ) the screening decision, and 2) the substantiation decision following an investigation by child protective services. The reports examined in this study $(n=720)$ are a representative sample of reports received by the Child and Youth Protection Centre of Quebec City (Quebec, Canada) in 1994-1995. Data collection consisted of a survey questionnaire completed by the caseworkers as well as information gathered in the children's files. On the whole, the child's security or development is considered compromised for a relatively low percentage of the reported children (17.2\%). Based on the results of logistic regression analyses, the best predictors of the outcome of the decisions are: the source of the report, the nature of allegations, the alleged perpetrator, the administrative unit which received the report and the case status in the agency. Similarities and differences are observed between the two decisions. The findings of the study point to a number of issues linked to the evaluation of reports and the orientation of the reported children.

\section{Introduction}

Les mauvais traitements envers les enfants recouvrent une large gamme de comportements de gravité diverse qui ne sont pas tous ni toujours ni partout reconnus comme des abus et qui ne suscitent pas toujours ni partout le même type de «traitement » par la société. Il en est de même des problèmes de comportement des jeunes qui sont fréquemment associés, voire confondus, avec des problèmes d'abus ou de négligence parentale ou encore identifiés à la délinquance. Il est dès lors permis de se demander comment, dans un contexte social et historique donné, se définissent ces problèmes et quels sont les modes d'intervention auxquels ils donnent lieu.

S'inscrivant dans la perspective de la réaction ou de la définition sociale $^{1}$, notre recherche porte sur la désignation institutionnelle des

1. Cette perspective met l'accent non pas sur les causes des situations-problèmes mais plutôt sur le processus par lequel se construisent les définitions des problèmes sociaux et sur ceux qui ont le pouvoir de définir les situations comme étant problématiques. Dans le domaine des abus et mauvais traitements envers les enfants, mentionnons notamment les travaux de Pfhol, 1977 ; Gelles, 1979 ; Giovannoni et Becerra, 1979 ; Parton, 1979 ; Gordon, 1988 ; Manseau, 1990 ; Robin, 1991 ; Thorpe, 1994). 
enfants « en besoin de protection » dans la société québécoise actuelle. Nous nous intéressons à la définition donnée aux situations signalées au sein des services de protection de la jeunesse et aux différents facteurs qui influencent les décisions prises par les intervenants. En raison de la portée de ces décisions et des enjeux qu'elles comportent non seulement pour les enfants signalés et leurs familles mais aussi pour l'ensemble des ressources concernées par les problèmes de jeunes en difficulté, ces questions comportent à notre avis un intérêt indéniable.

$\mathrm{Au}$ Québec, c'est dans le cadre de la Loi sur la protection de la jeunesse que sont déterminées les situations ${ }^{2}$ en vertu desquelles « la sécurité ou le développement d'un enfant » sont considérés comme étant ou pouvant être compromis. Ces situations sont généralement regroupées en cinq grandes problématiques : abandon, négligence, incluant le rejet affectif grave, abus physiquess, abus sexuelss et troubles sérieux du comportement. Notons ainsi que la législation québécoise s'applique à des situations mettant en cause non seulement les comportements d'adultes vis-à-vis des enfants de moins de 18 ans mais également les comportements des jeunes eux-mêmes.

Deux étapes du processus d'intervention en protection de la jeunesse ont été étudiées : la réception et l'évaluation des signalements. C'est essentiellement à travers ces deux étapes que les intervenants déterminent si la situation de l'enfant nécessite une prise en charge par les services de protection. Lors de la première étape, les intervenants effectuent un premier filtrage des plaintes reçues. À l'issue de cette étape, le signalement peut être retenu ou non pour évaluation. S'il n'est pas retenu, il peut être orienté vers une autre ressource du milieu. La deuxième étape consiste en une investigation plus approfondie de la situation signalée nécessitant une rencontre avec l'enfant, ses parents et toute autre personne jugée significative. Cette évaluation donne lieu à une décision statuant sur la compromission de la sécurité ou du développement de l'enfant. Trois issues sont possibles : 1) les faits sont jugés fondés et l'enfant est considéré en besoin de protection ; 2) les faits sont jugés fondés sans toutefois que l'enfant ne soit considéré en besoin de protection ; 3) les faits sont jugés non fondés. Dans ces deux derniers cas, il y a fermeture du dossier avec ou sans référence.

Notre analyse poursuivait trois objectifs :1) décrire les trajectoires des enfants signalés aux étapes de réception et d'évaluation des signa-

2. Ces situations sont énumérées aux articles 38 et 38.1 de la LPJ. 
lements ; 2) identifier les facteurs qui influencent la décision de retenir le signalement et la décision sur la compromission ; 3) comparer ces facteurs pour chacune des décisions rendues.

\section{Recension des écrits}

Plusieurs travaux de recherche ont porté sur les facteurs qui influencent la prise de décision aux étapes de réception et d'évaluation des signalements. Sans reprendre de façon détaillée les résultats de ces recherches et en gardant en mémoire les problèmes de comparaison (Zuravin et al., 1995 ; Jacob et Laberge, 1997) qu'elles comportent ${ }^{3}$, nous relèverons les principaux facteurs ayant retenu l'attention jusqu'ici. Ceux-ci ont été regroupés en six grandes catégories selon qu'ils se rapportent : 1) à la situation signalée ; 2) au signalant ou source du signalement ; 3) à l'enfant signalé ; 4) aux parents et à la structure familiale ; 5) aux ressources du milieu ; et enfin 6) à la structure d'intervention elle-même.

1) Parmi les caractéristiques de la situation, mentionnons en premier lieu le type de problème rapporté. Ces problèmes doivent être perçus comme relevant du mandat légal de l'établissement et définis comme des situations d'abus ou de négligence (Giovannoni, 1991). Des allégations d'abus physiquess et surtout d'abus sexuelss accroissent la probabilité que le signalement soit retenu lors du premier screening (Hutchison, 1989 ; Wells et al., 1991 ; 1995 ; Jones, 1996). Ces deux types d'abus sont aussi plus fréquemment jugés fondés (Groeneveld et Giovannoni, 1977 ; Eckenrode et al., 1988b ; Drake, 1995) ou évalués comme des cas de protection (Jones, 1996) que les situations de négligence, les résultats obtenus par Trocmé et al. (1994) faisant figure d'exception. Dans l'étude de Hutchison (1989), seules des allégations d'abus physiques augmentent la probabilité que le signalement soit jugé fondé. La présence de blessures ou d'évidence physique d'abus ou encore le fait que la situation ait exigé l'hospitalisation de l'enfant (DiLeonardi, 1980 ; Rosen, 1981 ; Hutchison, 1989 ; Giovannoni, 1989, 1991 ; Trocmé et al., 1994 ; Wells et al., 1995 ; Smith et al., 1995 ; Deisz et al.,

3. Ces études réferent en effet à des contextes forts divers sur le plan légal et administratif. Les situations d'abus ou de mauvais traitements qui sont considérées different également selon les recherches. Enfin, on observe des disparités importantes sur le plan méthodologique, tant au niveau de la taille des échantillons, des méthodes de collecte des données, des variables étudiées et de leur opérationnalisation que des techniques d'analyse utilisées par les chercheurs. 
1996), la perception de conséquences psychologiques de même que la durée (Trocmé et al., 1994 ; Smith et al., 1995), le nombre d'incidents et de types de mauvais traitements rapportés (Giovannoni, 1989) sont d'autres éléments qui influent sur les jugements portés par les intervenants. Le nombre d'enfants signalés constitue également une caractéristique relevée dans les recherches (DiLeonardi, 1980 ; Wells et al., 1995 ; Zuravin et al., 1995 ; Drake, 1996a) bien que son influence ne s'avère pas toujours significative. Il en est de même concernant le fait d'avoir déjà été l'objet d'un signalement (Hawkins et Duncan, 1985 ; Eckenrode et al., 1988a ; Hutchison, 1989 ; Winefield et Bradley, 1992; Trocmé et al., 1994 ; Haskett et al., 1995 ; Wells et al., 1995). Plusieurs études ayant porté sur les politiques de screening ou sur les signalements montrent également que l'identité de la personne mise en cause influence les décisions de retenir le signalement (Downing et al., 1990 ; Wells et al., 1989, 1995) et d'en déterminer le bien-fondé (DiLeonardi, 1980 ; Trocmé et al., 1994 ; Haskett et al.,1995 ; Zuravin et al., 1995).

Les caractéristiques de la situation réferent en outre aux jugements portés par les intervenants sur les faits : gravité, précision et validité des informations recueillies. Le caractère de gravité imputé à la situation (Giovannoni, 1989, 1991 ; Winefield et Bradley, 1992 ; Trocmé et al., 1994 ; Zuravin et al., 1987, 1995) et l'évaluation du risque par l'intervenant (Smith et al., 1995) se révèlent des facteurs clés permettant de prédire si le signalement sera fondé ou non. Le fait qu'aucun acte spécifique ne soit allégué ou encore l'absence d'informations suffisantes sont également des raisons fréquemment invoquées par les intervenants pour ne pas retenir un signalement (Giovannoni, 1991 ; Wells et al., 1991). Il s'agit de critères souvent énoncés dans les politiques de screening existantes (Wells et al., 1989 ; Downing et al., 1990). La précision des faits et la qualité de la preuve disponible déterminent également l'issue de l'évaluation (Giovannoni, 1991). La crédibilité accordée au signalant joue par ailleurs un rôle important dans la prise de décision (Downing et al., 1990 ; Wells et al., 1989, 1991 ; Giovannoni, 1991). Outre le témoignage du déclarant, ceux de l'enfant signalé et de ses parents sont aussi pris en considération. En l'absence de preuves tangibles d'abus, la crédibilité accordée à leur version des faits acquiert une importance accrue (Waterhouse et Carnie, 1992 ; Jones, 1993 ; Haskett et al., 1995).

2) La source du signalement est sans contredit la caractéristique qui a été le plus souvent étudiée dans les recherches ayant porté sur le processus décisionnel dans les services de protection de la jeunesse 
(Zuravin et al., 1995). Selon la plupart de ces recherches, il existe une distinction très nette entre les signalements provenant des déclarants professionnels et non professionnels (Groeneveld et Giovannoni, 1977 ; DiLeonardi, 1980 ; Eckenrode et al., 1988a, 1988b ; Flango, 1991 ; Winefield et Bradley, 1992 ; Trocmé et al., 1994 ; Drake, 1995 ; Wells et al., 1995 ; Zuravin et al., 1987, 1995). Il ressort en outre que les signalements anonymes sont généralement ceux qui sont le moins souvent jugés fondés (Adams et al., 1982 ; Zuravin et al., 1987 ; Flango, 1991 ; Trocmé et al., 1994 ; Drake, 1995 ; Eckenrode et al., 1998a).

Certaines recherches ont par ailleurs tenté de mettre en évidence des différences plus fines entre les catégories de déclarants. Les policiers figurent habituellement parmi les signalants ayant le plus de chances de voir leurs signalements jugés fondés (Groeneveld et Giovannoni, 1977 ; Eckenrode et al., 1988a ; Winefeld et Bradley, 1992 ; Trocmé et al., 1994 ; Drake, 1995). Les résultats sont moins clairs concernant les autres catégories de signalants professionnels. Par ailleurs, s'il est vrai que les signalements des professionnels sont plus fréquemment jugés fondés, cette constatation d'ensemble masque des exceptions importantes concernant certaines sous-catégories de déclarants non professionnels (Eckenrode et al., 1988a, 1988b ; Hutchison, 1989 ; Winefield et Bradley, 1992 ; Trocmé et al., 1994 ; Wells et al., 1995).

3) Les caractéristiques de l'enfant signalé apparaissent également au cœur du processus décisionnel. L'âge constitue l'indicateur par excellence du degré de vulnérabilité de l'enfant. Les recherches ayant examiné l'influence de ce facteur présentent néanmoins des résultats divergents (Eckenrode et al., 1988a, 1988b ; Hutchison, 1989 ; Winefield et Bradley, 1992 ; Trocmé et al., 1994 ; Haskett et al., 1995 ; Smith et al., 1995 ; Wells et al., 1995 ; Zuravin et al., 1995 ; Freeman et al., 1996). Selon Eckenrode et al. (1988b), les résultats contradictoires obtenus dans les recherches s'expliquent possiblement par le fait que l'influence de l'âge s'exerce différemment selon le type de situation signalée. Quelques études mettent par ailleurs en évidence l'influence du sexe de l'enfant signalé sur les décisions prises par les intervenants (Eckenrode et al., 1988a ; Wells et al., 1995). D'après plusieurs recherches toutefois (Hutchison, 1989 ; Winefield et Bradley, 1992 ; Haskett et al., 1995 ; Zuravin et al., 1995 ; Freeman et al., 1996), le sexe de l'enfant ne s'avère pas avoir d'impact sur les décisions de retenir et/ou de juger le signalement fondé. L'origine ethnique est également une caractéristique qui a suscité l'intérêt de nombreux chercheurs. Comme le 
souligne Jones (1993 : 249) dans sa recension des écrits : « Ethnicity is a variable where conflicting findings at all choice points in the literature are generally the rule. » Les recherches ayant porté sur la réception et l'évaluation des signalements ne font pas exception (Kotch et Thomas, 1986 ; Eckenrode et al., 1988a, 1988b ; Hutchison, 1989 ; Winefield et Bradley, 1992 ; Tourigny et Bouchard, 1994 ; Wells et al., 1995 ; Zuravin et al., 1995 ; Drake, 1996a). Le comportement de l'enfant, son développement physique, intellectuel, social et affectif, tout comme sa réaction vis-à-vis de sa situation, de ses parents et du signalement influencent également le jugement porté par les travailleurs sociaux et le type d'intervention recommandé (DiLeonardi, 1980 ; Rosen, 1981 ; Waterhouse et Carnie, 1992 ; Jones, 1993 ; Shapira et Benbenishty, 1993 ; Haskett et al., 1995 ; Smith et al., 1995). L'absence de corroboration des faits par l'enfant ou, à l'inverse, des allégations spécifiques de sa part se révèlent en outre de première importance (Giovannoni, 1991 ; Waterhouse et Carnie, 1992 ; Haskett et al., 1995 ; Smith et al., 1995).

4) Une quatrième catégorie de facteurs se rapporte aux caractéristiques des parents et, plus généralement, aux caractéristiques de la famille de l'enfant signalé. Certaines recherches ont ainsi exploré l'influence de facteurs associés à la « capacité parentale ». Ces facteurs sont nombreux et réferrent à la fois aux dimensions matérielles, morales, intellectuelles et affectives de l'exercice du rôle parental : habiletés déficientes concernant les soins à donner aux enfants, problèmes de santé mentale ou physique, difficultés conjugales ou familiales, problèmes de consommation d'alcool ou de drogue, déviance élevée par rapport aux normes existantes... (DiLeonardi, 1980 ; Rosen, 1981 ; Alter, 1985 ; Hutchison, 1989 ; Waterhouse et Carnie, 1992 ; Jones, 1993 ; Shapira et Benbenisty, 1993 ; Smith et al., 1995). La capacité parentale fait également référence à l'attitude des parents vis-à-vis de la situation (Giovannoni, 1991 ; Waterhouse et Carnie, 1992 ; Smith et al., 1995) de même qu'à leur degré de motivation et de collaboration avec les intervenants (Alter, 1985 ; DiLeonardi, 1980 ; Waterhouse et Carnie, 1992 ; Shapira et Benbenishty, 1993). Le fait de reconnaître la situation ou encore de collaborer avec les services témoignent d'une volonté de changement de la part des parents, réduisant du même coup les risques pour l'enfant.

En ce qui a trait au statut socio-économique, de nombreuses études, au Québec comme ailleurs, ont de surcroît mis en évidence la surreprésentation des familles de faible niveau socio-économique parmi les familles prises en charge par les services de protection de la jeunesse. La 
question qui nous intéresse cependant ici est de savoir s'il s'opère une sélection en regard du statut-économique à l'intérieur même des services de protection, question qui n'a pas encore trouvé de réponse définitive (Manseau, 1990 ; Winefield et Bradley, 1992 ; Trocmé et al., 1994 ; Callahan et Lumb, 1995 ; Drake et Pandy, 1996). Le même questionnement s'applique à l'endroit des familles monoparentales et recomposées. Kotch et Thomas (1986) n'observent aucune association entre le type de famille et le jugement porté sur le bien-fondé du signalement. Hawkins et Duncan (1985) relèvent cependant que les signalements des enfants appartenant à des familles « brisées » (broken families) sont moins souvent jugés fondés. Trocmé et al. (1994) constatent pour leur part que les familles monoparentales à chef féminin présentent le pourcentage le plus faible de signalements fondés et les familles recomposées, le pourcentage le plus élevé.

5) Parmi les déterminants du processus décisionnel au sein des services de protection de la jeunesse, soulignons, en cinquième lieu, le rôle joué par les ressources existantes, désigné dans le langage administratif comme la « capacité du milieu ». Concrètement, cette « capacité » fait référence aux ressources qui peuvent être mobilisées (ou qui le sont déjà) auprès de l'enfant signalé et de ses parents, permettant d'éviter que leur situation ne soit prise en charge par les services de protection. Il peut s'agir du soutien offert par des ressources formelles (établissements de services de santé ou de services sociaux, milieu scolaire, organismes communautaires) ou encore par des ressources informelles telles la parenté, les amis ou le voisinage. Peu de recherches ont toutefois tenté de mesurer l'influence des ressources du milieu sur les décisions prises durant les premières étapes du processus d'intervention (Wells et al., 1989 ; Downing et al., 1990). Le même constat peut être formulé à l'égard des ressources informelles et de la qualité du soutien social dont bénéficient les parents (Kotch et Thomas, 1986).

6) La dernière catégorie de facteurs que nous avons identifiée réfère à la structure d'intervention elle-même. Aux États-Unis, les études de Wells et al. $(1991,1995)$ montrent qu'il existe des différences notables d'un établissement à l'autre en ce qui a trait aux politiques et aux pratiques entourant le traitement des signalements à l'entrée des services de protection. D'autres études observent des variations dans la proportion de signalements jugés fondés selon les établissements (Hutchison, 1989 ; Trocmé et al. 1994). Wolock (1982) met pour sa part en relief l'influence du « caseload » sur l'évaluation. Les caractéristiques liées 
à l'organisation du travail et au fonctionnement des établissements peuvent également avoir un impact sur les décisions prises par les intervenants tout comme les relations entre intervenants et entre catégories professionnelles (Deleury et al., 1985 ; Manseau, 1990) et comme les caractéristiques personnelles ou professionnelles des intervenants euxmêmes (Hutchison, 1989 ; Manseau, 1990 ; Giovannoni, 1991 ; Trocmé et al. 1994).

Il ressort de la recension des écrits que les facteurs susceptibles d'influencer les décisions prises par les intervenants sont nombreux. Étant donné le peu d'études sur le sujet au Québec ${ }^{4}$, nous avons opté pour une approche globale ne se limitant ni à une problématique particulière ni à une seule catégorie de déterminants. Parmi l'ensemble des facteurs relevés dans la littérature, une attention particulière a néanmoins été accordée aux caractéristiques de la situation signalée et à la source du signalement. L'impact des facteurs se rapportant à la structure d'intervention n'a pas été examiné dans notre étude. Ajoutons que très peu de recherches (Hutchison, 1989 ; Jones, 1996) se sont penchées à la fois sur les étapes de screening et d'évaluation des signalements et ont tenté d'en comparer les logiques respectives.

\section{Méthodologie}

Site de la recherche et échantillon

La recherche s'est déroulée au Centre jeunesse de Québec qui dessert la population résidant sur l'ensemble du territoire de la région administrative de Québec (région 03). Il s'agit d'une population majoritairement urbaine, fortement homogène sur le plan ethnique, linguistique et culturel.

Tous les signalements reçus au centre jeunesse entre le 5 décembre 1994 et le 5 février 1995 ont été étudiés, soit un total de 720 signalements. Cette méthode a été utilisée en raison de l'impossibilité d'avoir recours aux techniques d'échantillonnage probabilistes usuelles. Au

4. Deux études d'envergure sont cependant en cours actuellement: The Canadian Incidence Study of Reported Child Abuse and Neglect (CIS), sous la direction de Nico Trocmé et l'Étude sur l'incidence et les caractéristiques des situations d'abus, de négligence, d'abandon et de troubles de comportement sérieux signalées à la Direction de la protection de la jeunesse au Québec (ÉIQ), dirigée par Marc Tourigny, Micheline Mayer et John Wright. Les deux études sont menées en parallèle. Un volet de l'étude québécoise s'inscrit dans l'étude canadienne. 
moment de l'étude en effet, la grande majorité des signalements non retenus étaient détruits dans les jours suivant la décision rendue. Certaines données sur ces signalements étaient cependant colligées de façon systématique par l'établissement, ce qui nous a permis d'examiner, a posteriori, la représentativité de l'échantillon. Pour ce faire, des analyses de chi ${ }^{2}$ ont été effectuées sur les variables suivantes : 1) la catégorie de signalants ; 2) l'unité administrative ayant reçu le signalement ; 3) le mode de contact avec l'établissement ; 4) la décision rendue à l'étape de la réception des signalements ; 5) la référence effectuée lorsque le signalement n'est pas retenu ; 6) la suite donnée au signalement retenu. Les analyses de $\mathrm{chi}^{2}$ montrent que l'échantillon est représentatif des signalements reçus au Centre jeunesse de Québec pour l'année administrative 1994-95 $(\mathrm{N}=4842)$, à l'exception de l'unité administrative ayant reçu le signalement ${ }^{5}$.

\section{Méthode de collecte} et d'analyse des données

Diverses modalités ont été utilisées pour la collecte des données. À l'étape de la réception des signalements, les données ont été recueillies à partir des formulaires déjà en usage au centre jeunesse (fiches de signalement non retenu) et à partir d'un court questionnaire conçu avec les intervenants. À l'étape de l'évaluation des signalements, ce sont les renseignements contenus dans les fiches de signalement retenu et dans les rapports d'évaluation, consignés aux dossiers des enfants, qui constituent nos sources d'information.

Afin d'identifier les facteurs qui influencent les deux décisions étudiées, des analyses de régression logistique ont été effectuées. Dans ces analyses, la méthode de sélection des variables est celle proposée par Hosmer et Lemeshow (1989 : 83-91). La première étape consiste à examiner séparément l'influence de chacune des variables indépendantes sélectionnées sur la variable dépendante. En second lieu, toutes les variables qui présentent un certain degré d'association avec la variable dépendante sont intro-

5. Le service régulier reçoit les demandes de services et les signalements aux heures habituelles d'ouverture du centre jeunesse. Le service d'urgence sociale répond à ces demandes en dehors des heures régulières (soir, nuit, fins de semaine, jours fériés). Dans notre étude, les signalements en provenance de l'Urgence sociale représentent $36 \%$ des signalements reçus comparativement à 17,5\% selon les données fournies par l'établissement pour l'année administrative 1994-1995. Le fait que l'étude ait été réalisée durant la période des Fêtes explique en partie, à notre avis, l'écart observé. 
duites dans le modèle. L'importance de chacune de ces variables est par la suite examinée et les variables qui ne contribuent pas au modèle sont éliminées. La dernière étape consiste à considérer la possibilité d'interactions entre les variables indépendantes. Dans notre recherche toutefois, nous n'avons pas examiné les interactions en raison de la présence d'un trop grand nombre de « cellules vides » qui se traduit notamment par des erreurs standard extrêmement élevées et qui ne permet pas d'estimer adéquatement les paramètres du modèle (Hosmer et Lemeshow, 1989 : 126-129 ; Menard, 1995 : 67-69)

Pour l'étude des facteurs influençant la décision sur la compromission (variable nominale polytomique), nous avons eu recours à l'analyse de régression logistique selon des modalités un peu différentes de celles ayant été utilisées pour l'étude des facteurs influençant la décision de retenir le signalement (variable nominale dichotomique ). Après avoir choisi le modèle permettant le mieux de prédire la décision rendue à l'aide d'une régression polytomique, nous avons réalisé séparément deux régressions dichotomiques. Ces analyses comparent les signalements pour lesquels la compromission a été établie aux signalements dont les faits ont été jugés non fondés, d'une part, et aux signalements pour lesquels les faits ont été jugés fondés mais la compromission non établie, d'autre part ${ }^{6}$.

Variables à l'étude et caractéristiques de l'échantillon

Parmi les multiples facteurs ou déterminants susceptibles d'influencer les décisions des intervenants, quatorze ont été examinés dans les analyses de régression logistique (tableau 1). Ces facteurs réferent plus généralement à quatre grandes catégories de déterminants : 1) les caractéristiques reliées à la situation signalée et au traitement administratif des signalements ; 2) les caractéristiques du signalant ; 3) les caractéristiques de l'enfant signalé et de son milieu de vie ; et, 4) les caractéristiques des ressources impliquées auprès de l'enfant ou de sa famille au moment du signalement.

6. Différentes mesures permettent par ailleurs de porter un jugement d'ensemble sur le modèle choisi et d'en évaluer l'ajustement (fit) aux données. Nous avons principalement utilisé le chi ${ }^{2} \mathrm{du}$ modèle (model chi-square), la déviance (likelihood ratio) et le $\mathrm{R}_{\mathrm{L}}^{2}$ proposés par Hosmer et Lemeshow (1989) (voir aussi Menard, 1995 et Agretsi, 1990). Idéalement, on souhaite obtenir un modèle dans lequel le $\mathrm{chi}^{2} \mathrm{du}$ modèle est statistiquement significatif $(p<0,05)$, la déviance, statistiquement non significative $(p>0,05)$ et le $R_{L}^{2}$, élevé. 
TA B LEA U 1

Variables à l'étude et caractéristiques de l'échantillon

\begin{tabular}{|c|c|c|c|c|}
\hline \multicolumn{5}{|l|}{ I- VARIABLES INDÉPENDANTES } \\
\hline & \multicolumn{4}{|c|}{ Étapes du processus d'intervention } \\
\hline & \multicolumn{2}{|c|}{$\begin{array}{l}\text { Réception des } \\
\text { signalements }\end{array}$} & \multicolumn{2}{|c|}{$\begin{array}{c}\text { Évaluation des } \\
\text { signalements }\end{array}$} \\
\hline & $\mathbf{n}$ & $\%$ & $\mathbf{n}$ & $\%$ \\
\hline \multicolumn{5}{|l|}{$\begin{array}{l}\text { 1- Caractéristiques reliées à la } \\
\text { situation et au traitement } \\
\text { administratif des signalements }\end{array}$} \\
\hline \multicolumn{5}{|l|}{ 1. Problématique identifiée } \\
\hline 1. Négligence/abandon & 331 & 48,0 & 143 & 53,0 \\
\hline 2. Troubles de comportement & 169 & 24,5 & 65 & 24,1 \\
\hline 3. Absentéisme scolaire & 30 & 4,3 & 4 & 1,5 \\
\hline 4. Abus sexuel & 61 & 8,8 & 25 & 9,3 \\
\hline 5. Abus physique & 75 & 10,9 & 33 & 12,2 \\
\hline 6. Conflit familial et autres & 24 & 3,5 & - & - \\
\hline Données manquantes & 30 & & & \\
\hline \multicolumn{5}{|l|}{ 2. Personne mise en cause } \\
\hline 1. Père & 96 & 14,3 & 35 & 13,0 \\
\hline 2. Mère & 203 & 30,2 & 86 & 31,9 \\
\hline 3. Enfant signalé & 160 & 23,8 & 50 & 18,5 \\
\hline 4. Conjoint/parenté & 34 & 5,1 & 15 & 5,5 \\
\hline 5. Tiers & 33 & 4,9 & 3 & 1,1 \\
\hline 6. Plus d'une personne & 147 & 21,8 & 81 & 30,0 \\
\hline Données manquantes & 47 & & & \\
\hline \multicolumn{5}{|l|}{ 3. Nombre d'enfants signalés } \\
\hline 1 & 454 & 63,2 & 172 & 63,6 \\
\hline 2 & 150 & 20,9 & 52 & 19,3 \\
\hline 3 & 57 & 7,9 & 21 & 7,8 \\
\hline 4 et + & 57 & 7,9 & 25 & 9,3 \\
\hline Données manquantes & 2 & & & \\
\hline \multicolumn{5}{|l|}{$\begin{array}{l}\text { 4. Unité administrative ayant } \\
\text { reçu le signalement }\end{array}$} \\
\hline 1. Urgence sociale & 260 & 36,1 & 101 & 37,4 \\
\hline 2. Accueil & 460 & 63,9 & 169 & 62,6 \\
\hline \multicolumn{5}{|l|}{$\begin{array}{l}\text { 5. Statut de l'enfant } \\
\text { au Centre jeunesse }\end{array}$} \\
\hline 1. Nouveau & - & - & 159 & 58,9 \\
\hline 2. Actif & - & - & 40 & 14,8 \\
\hline 3. Classé & - & - & 71 & 26,3 \\
\hline \multicolumn{5}{|l|}{ 6. Priorité d'intervention } \\
\hline 1. Immédiat & - & - & 74 & 27,4 \\
\hline 2. 24 heures & - & - & 57 & 21,1 \\
\hline 3.4 jours & - & - & 139 & 51,5 \\
\hline
\end{tabular}


TABLEA U 1

Variables à l'étude et caractéristiques de l'échantillon (suite)

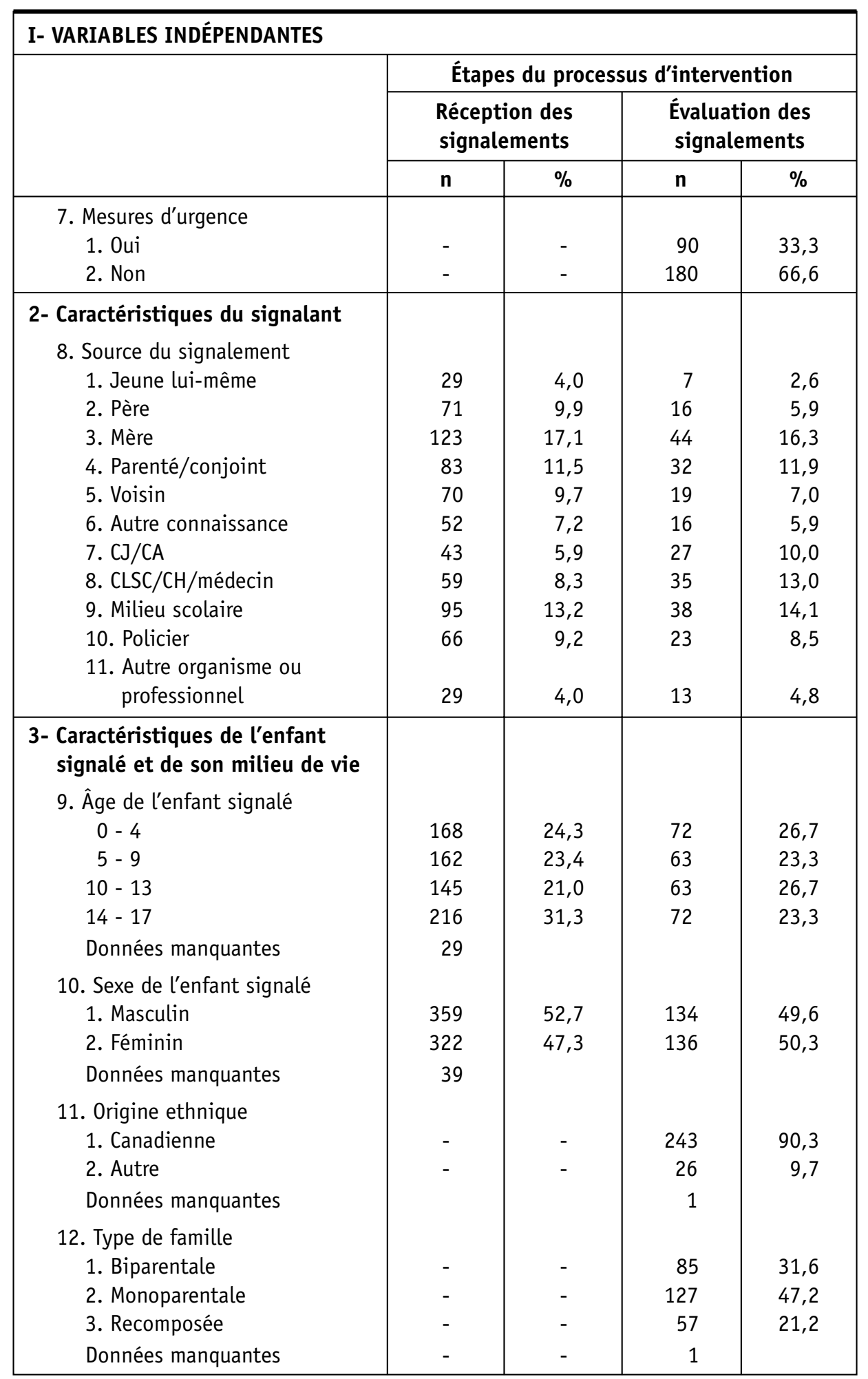


TABLEA U 1

Variables à l'étude et caractéristiques de l'échantillon (suite II)

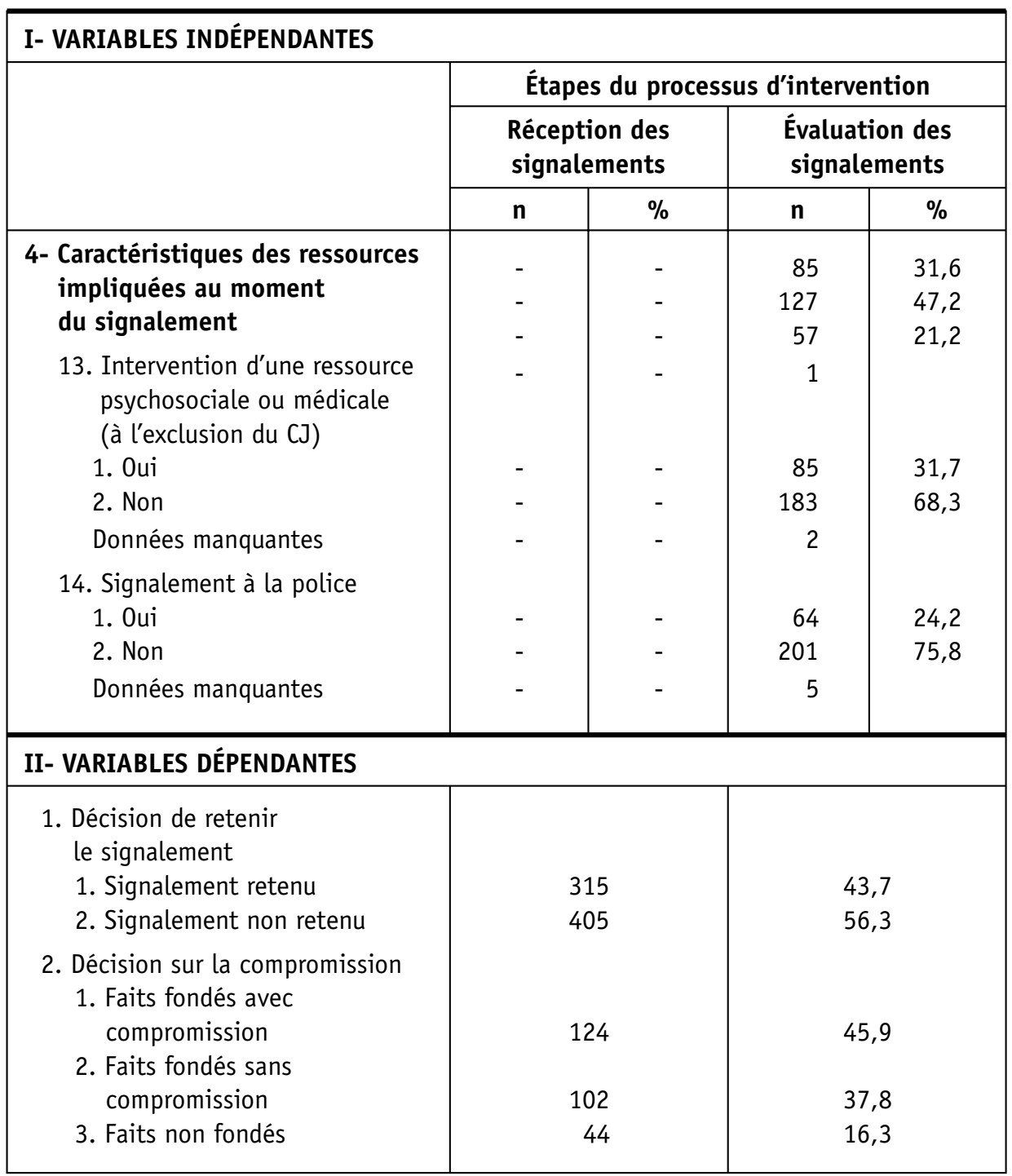

Parmi ces variables, sept n'ont été considérées qu'à l'étape de l'évaluation parce qu'elles étaient spécifiques aux signalements retenus (priorité d'intervention, mesures d'urgence) ou encore parce qu'on ne disposait pas d'informations suffisamment complètes à l'étape de la réception des signalements (origine ethnique de l'enfant signalé, statut dans l'établissement, type de famille, intervention d'une ressource psychosociale ou 
médicale, signalement à la police) ${ }^{7}$. Les caractéristiques de l'échantillon, à chacune des étapes étudiées, sont présentées au tableau 1 .

\section{Résultats}

Les trajectoires des signalements et des enfants signalés

Sans faire état de manière détaillée des trajectoires des enfants signalés, indiquons néanmoins qu'à chaque étape du processus d'intervention des constats similaires peuvent être effectués. Premier constat : une proportion importante d'enfants voient leur signalement non retenu ou leur dossier fermé à la suite des décisions rendues par les intervenants. Dans l'ensemble, la compromission est établie pour une fraction relativement limitée des enfants signalés (17,2\%) (figure 1). Second constat : des problèmes sont néanmoins identifiés dans un grand nombre de situations ne donnant pas lieu à une prise en charge par les services de protection, d'où la distinction établie et maintes fois invoquée par les intervenants entre les enfants en «besoin d'aide » et les enfants en «besoin de protection». Une proportion importante d'enfants sont ainsi dirigés vers d'autres ressources du milieu, majoritairement vers des ressources psychosociales mais aussi judiciaires, médicales et communautaires. Au total, environ $40 \%$ des signalements donnent lieu à une référence.

Ces résultats renvoient à la question centrale de la recherche : quels sont les facteurs qui influencent les décisions prises par les intervenants et qui peuvent rendre compte des diverses trajectoires suivies par les enfants signalés?

Les facteurs qui permettent de prédire la décision de retenir le signalement

Considérées séparément, cinq des sept variables indépendantes étudiées présentaient une association statistiquement significative $(p<0,05)$ avec

7. Bien que sept variables aient été reprises d'une analyse à l'autre, mentionnons cependant que les catégories des variables utilisées ne sont pas toujours identiques. En effet, à l'étape de l'évaluation, nous avons dû procéder à des regroupements en raison des effectifs très restreints de certaines catégories. Ainsi, les signalements d'absentéisme scolaire et de troubles de comportement ont été regroupés de même que ceux impliquant des tiers, des conjoints et des membres de la parenté. Il en a été de même concernant les signalements effectués par les jeunes eux-mêmes qui ont été classés avec ceux des conjoints et des membres de la parenté. 


\section{FIG URE 1}

Ensemble des trajectoires des enfants signalés aux étapes de réception et d'évaluation des signalements

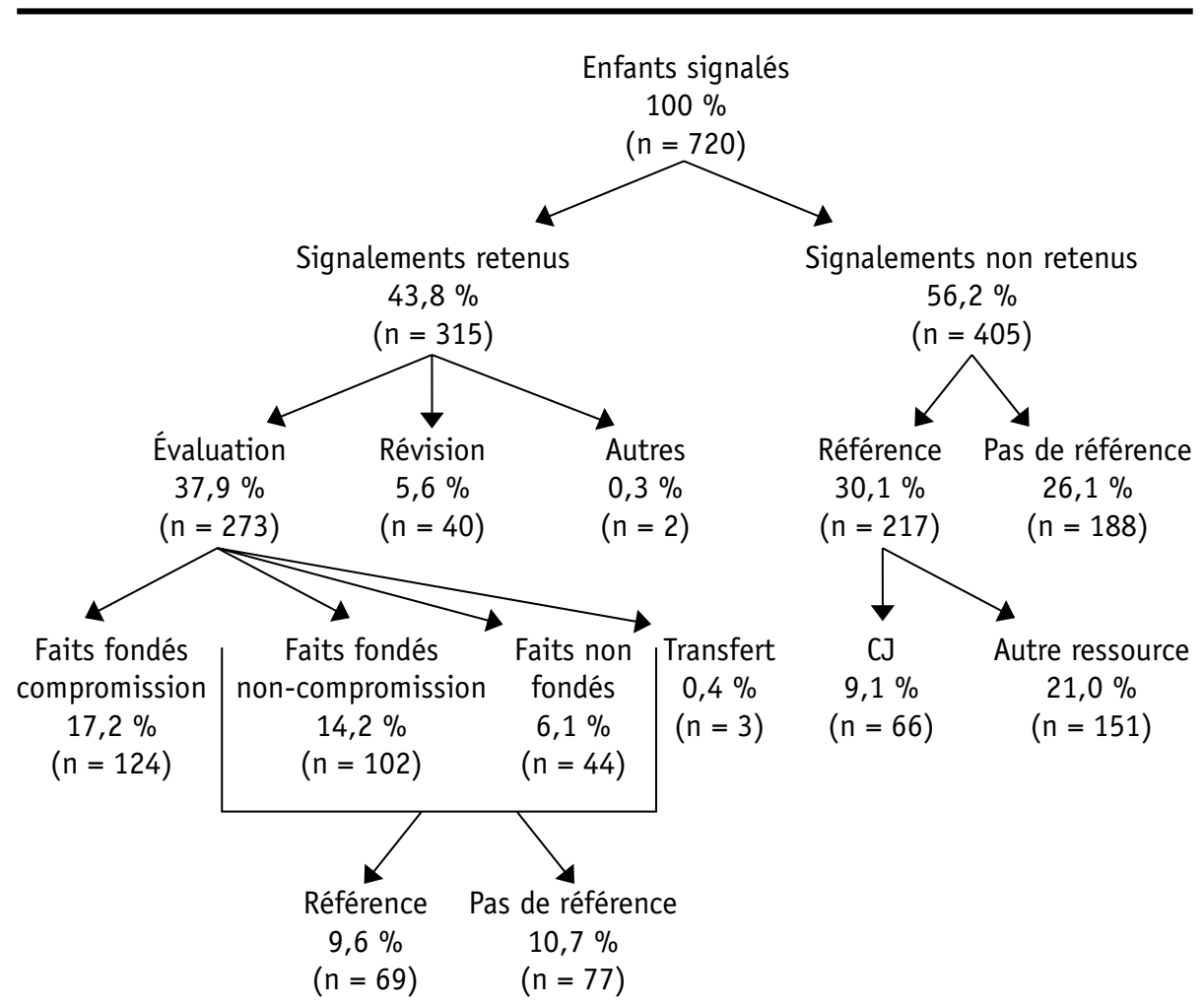

la décision de retenir le signalement. Seuls le sexe de l'enfant et le nombre d'enfants signalés n'étaient pas associés à la décision prise par les intervenants. Le tableau 2 expose les résultats de l'analyse de régression logistique multivariée. Selon le modèle retenu, quatre facteurs permettent de prédire la décision de retenir le signalement : la source du signalement, la problématique identifiée, la personne mise en cause et l'unité administrative ayant reçu le signalement. Ces quatre variables étant toutes des variables nominales, nous avons utilisé quatre groupes de variables indicatrices (dummy variables) (Hosmer et Lemeshow, 1989 : $45-46,48)$. Les catégories de référence choisies sont précisées au tableau 2.

En ce qui a trait au statut du déclarant, les résultats montrent que, si on tient compte de l'ensemble des variables incluses dans le modèle, ce sont les voisins, les connaissances, les membres de la parenté de même que les pères des enfants signalés qui présentent la probabilité la plus faible de voir leurs signalements retenus. Inversement, les chances qu'un signalement soit retenu sont les plus fortes lorsque le déclarant est un 
TA B LEA U 2

Résultats de la régression logistique quant à la décision de retenir le signalement (signalement retenu $=1$; signalement non retenu $=0)(n=672)$

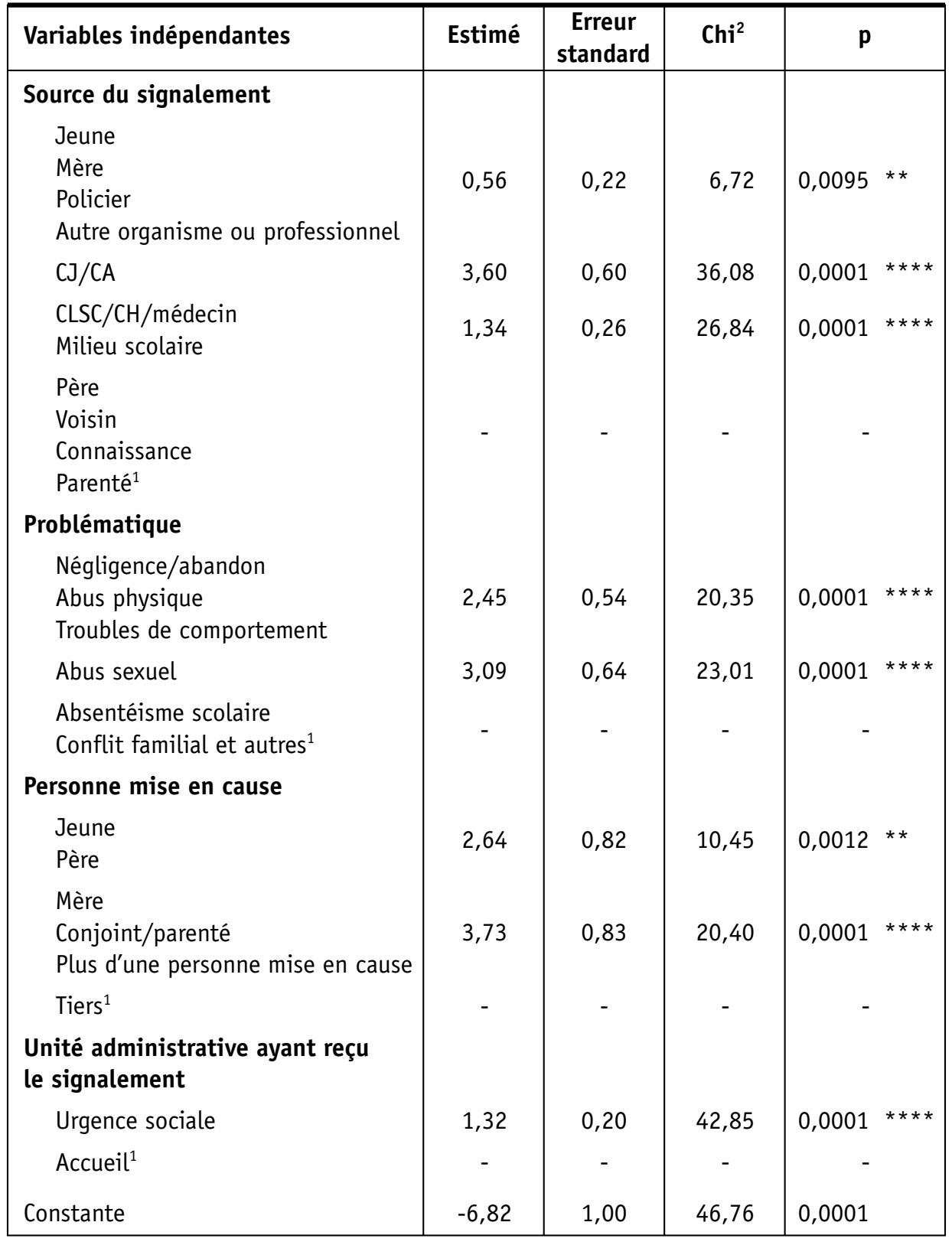

Chi ${ }^{2}$ du modèle : $186,23 \quad \mathrm{df}=8 \quad \mathrm{p}=0,0001$

Likelihood ratio : $39,38 \quad \mathrm{df}=35 \quad \mathrm{p}=0,2802$

$\mathrm{R}^{2} \mathrm{~L}: 82,5 \%$

${ }^{\star} \mathrm{p}<0,05^{\star \star} \mathrm{p}<0,01 \quad{ }^{\star \star \star} \mathrm{p}<0,001 \quad{ }^{\star \star \star \star} \mathrm{p}<0,0001$

1. Catégorie de référence 
intervenant œuvrant en centre jeunesse (CJ) ou en centre d'accueil (CA). Par ailleurs, les signalements pour absentéisme scolaire ou encore identifiés comme des conflits familiaux ont moins de chances d'être retenus que tous les autres types de problèmes signalés. Les signalements pour négligence, troubles de comportement et abus physiques ne se distinguent pas les uns des autres de façon significative alors que les signalements pour abus sexuels présentent une probabilité un peu plus élevée d'être retenus. Les résultats de l'analyse de régression logistique montrent également que la probabilité de voir les signalements impliquant un tiers retenus est plus faible que lorsqu'un membre de la famille ou de la parenté est mis en cause. Enfin, si on considère l'unité administrative ayant reçu le signalement, on constate que les signalements reçus par le service de l'urgence sociale ont plus de chances d'être retenus.

Les facteurs qui permettent de prédire la décision sur la compromission

Sept variables (nombre d'enfants signalés, priorité d'intervention, âge, sexe, origine ethnique de l'enfant signalé, type de famille, intervention d'une ressource médicale ou psychosociale) ne présentaient pas d'association statistique $(\mathrm{p}<0,05)$ avec la décision sur la compromission et ont par conséquent été exclues des analyses subséquentes.

Selon le modèle que nous avons retenu dans l'analyse de régression logistique polytomique (likelihood ratio $=141,31$; $\mathrm{df}=146$; $\mathrm{p}=0,59$ ), les quatre facteurs qui permettent le mieux de prédire la décision sur la compromission sont la source du signalement, la problématique identifiée, la personne mise en cause et le statut de l'enfant signalé. Les tableaux 3 et 4 font état des résultats des deux analyses de régression dichotomiques réalisées subséquemment. Encore une fois, les quatre variables explicatives retenues étant toutes des variables nominales, nous avons utilisé quatre groupes de variables indicatrices.

Les résultats de la première analyse de régression montrent que les voisins et les connaissances présentent, avec les intervenants classés dans la catégorie " autres », une probabilité plus forte de voir leurs signalements non fondés. À l'opposé, les parents, les policiers, les médecins, de même que les intervenants en CLSC, en milieu hospitalier et en milieu scolaire constituent les groupes pour lesquels cette probabilité est la plus faible. En second lieu, les chances qu'un signalement soit non fondé sont moins élevées lorsqu'il s'agit d'un problème de négligence ou de troubles de comportement. Les signalements d'abus sexuels sont ceux qui 
TAB LEAU 3

Résultats de la régression logistique quant à la décision sur la compromission (faits non fondés $=1$; faits fondés avec compromission $=0)(n=168)$

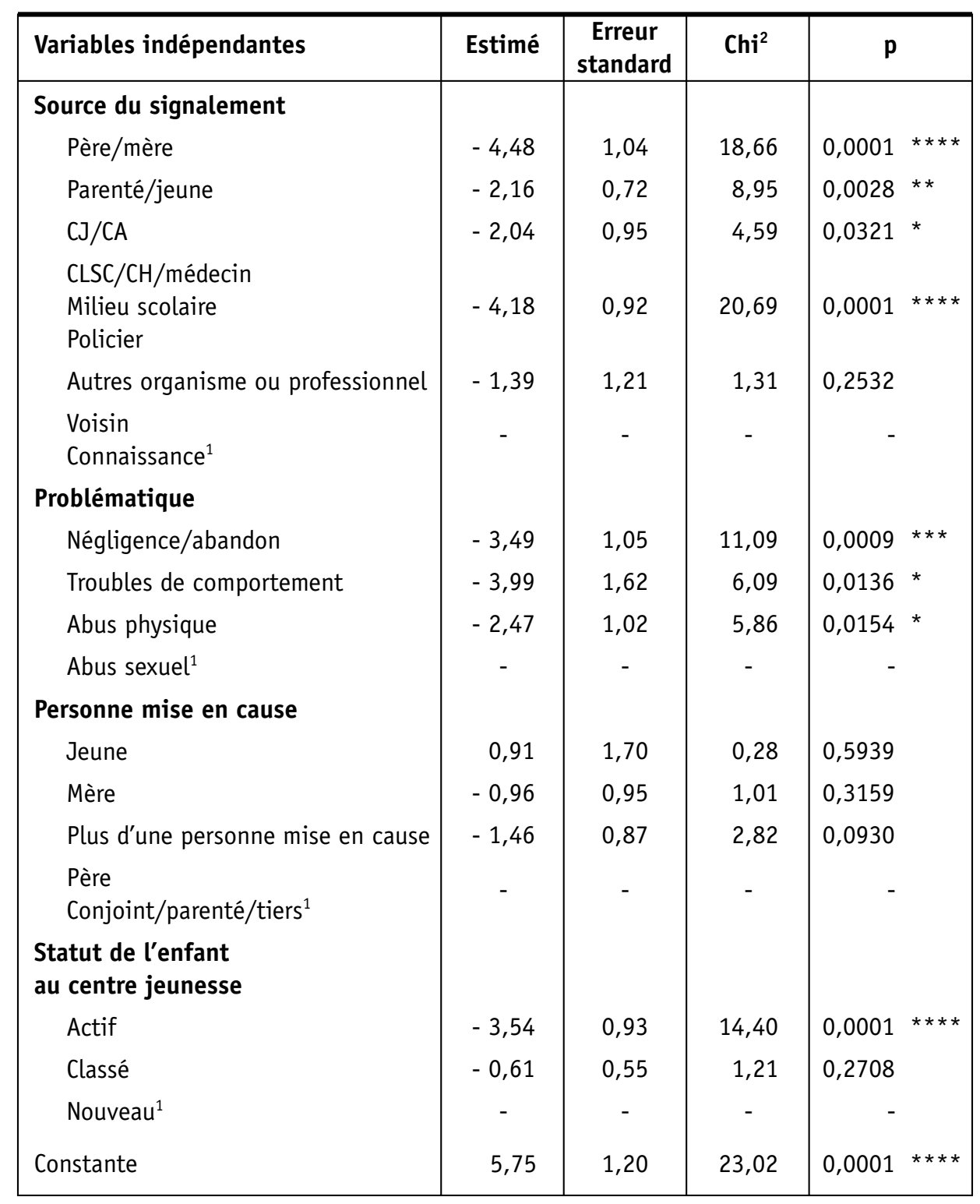

$\mathrm{Chi}^{2}$ du modèle : 81,26

Likelihood ratio : 69,80

$\mathrm{R}^{2} \mathrm{~L}: 53,8 \%$

${ }^{\star} \mathrm{p}<0,05 \quad \star \star \mathrm{p}<0,01$

df $=13 \quad \mathrm{p}=0,0001$

df $=56 \quad p=0,1017$

$\star \star \star \mathrm{p}<0,001 \quad \star \star \star \star \mathrm{p}<0,0001$

1. Catégorie de référence 
TABLEAU 4

Résultats de la régression logistique quant à la décision sur la compromission (faits fondés sans compromission $=1$; faits fondés avec compromission $=0)(n=226)$

\begin{tabular}{|c|c|c|c|c|}
\hline Variables indépendantes & Estimé & $\begin{array}{c}\text { Erreur } \\
\text { standard }\end{array}$ & $\mathrm{Chi}^{2}$ & $\mathrm{p}$ \\
\hline \multicolumn{5}{|l|}{ Source du signalement } \\
\hline Père/mère & $-0,18$ & 0,76 & 0,05 & 0,8164 \\
\hline Parenté/jeune & $-0,43$ & 0,81 & 0,28 & 0,5957 \\
\hline $\mathrm{CJ} / \mathrm{CA}$ & 0,61 & 0,88 & 0,47 & 0,4921 \\
\hline $\begin{array}{l}\text { CLSC/CH/médecin } \\
\text { Milieu scolaire } \\
\text { Policier }\end{array}$ & 0,23 & 0,72 & 0,10 & 0,7497 \\
\hline Autres organisme ou professionnel & 1,56 & 1,04 & 2,26 & 0,1328 \\
\hline $\begin{array}{l}\text { Voisin } \\
\text { Connaissance }{ }^{1}\end{array}$ & - & - & - & - \\
\hline \multicolumn{5}{|l|}{ Problématique } \\
\hline Négligence/abandon & $-0,42$ & 0,93 & 0,20 & 0,6553 \\
\hline Troubles de comportement & 0,26 & 1,08 & 0,06 & 0,8113 \\
\hline Abus physique & $-1,81$ & 1,04 & 3,05 & 0,0807 \\
\hline Abus sexuel $^{1}$ & - & - & - & - \\
\hline \multicolumn{5}{|l|}{ Personne mise en cause } \\
\hline Jeune & $-2,13$ & 0,88 & 5,81 & $0,0159 *$ \\
\hline Mère & $-2,10$ & 0,61 & 12,01 & $0,0005 * * *$ \\
\hline Plus d'une personne mise en cause & $-2,83$ & 0,63 & 20,10 & $0,0001 * * * *$ \\
\hline $\begin{array}{l}\text { Père } \\
\text { Conjoint/parenté/tiers }{ }^{1}\end{array}$ & - & - & - & - \\
\hline \multicolumn{5}{|l|}{$\begin{array}{l}\text { Statut de l'enfant } \\
\text { au centre jeunesse }\end{array}$} \\
\hline Actif & $-3,20$ & 0,67 & 22,73 & $0,0001 * * * *$ \\
\hline Classé & $-1,58$ & 0,38 & 17,57 & $0,0001 * * * *$ \\
\hline Nouveau $^{1}$ & - & - & - & - \\
\hline Constante & 2,77 & 1,17 & 5,62 & $0,0158 *$ \\
\hline
\end{tabular}

Chi $^{2}$ du modèle : $78,01 \quad \mathrm{df}=13 \quad \mathrm{p}=0,0001$

Likelihood ratio : 79,99 $\mathrm{df}=65 \quad \mathrm{p}=0,0997$

$\mathrm{R}_{\mathrm{L}}^{2}: 49,4 \%$

${ }^{\star} \mathrm{p}<0,05 \quad{ }^{\star \star} \mathrm{p}<0,01 \quad{ }^{\star \star \star} \mathrm{p}<0,001 \quad{ }^{\star \star \star \star} \mathrm{p}<0,0001$

1. Catégorie de référence 
présentent la probabilité la plus forte d'être non fondés, suivis par les signalements d'abus physiques. Enfin, les enfants n'ayant pas de dossier au centre jeunesse («nouveaux » enfants) détiennent également plus de chances de voir leurs signalements non fondés que les enfants ayant un dossier actif. L'influence de la personne mise en cause n'est pas statistiquement significative.

Comme le révèlent les résultats de la seconde analyse de régression (tableau 4), ici, c'est l'influence de la source du signalement et de la problématique identifiée qui ne s'avèrent pas significatives sur le plan statistique. Deux variables permettent principalement de prédire que la sécurité ou le développement de l'enfant ne sera pas jugé compromis lorsque les faits sont fondés : la personne mise en cause et le statut de l'enfant dans l'établissement. Si on considère les personnes mises en cause, lorsque les faits sont fondés, la probabilité que le dossier soit fermé à la suite de l'évaluation (pas de compromission) est plus élevée pour les signalements impliquant soit le père, soit un conjoint, un membre de la parenté ou un tiers. Les signalements mettant en cause plus d'un membre de la famille de même que ceux où les faits sont reprochés à la mère ou au jeune lui-même comportent moins de chances d'être fermés à la suite de l'évaluation. Le statut de l'enfant dans l'établissement permet également de prédire la décision qui sera rendue. À cet égard, ce sont les enfants ayant un dossier actif qui présentent la probabilité la plus faible de voir leur dossier fermé à la suite de l'évaluation, suivis des enfants ayant un dossier classé.

En résumé, quatre facteurs permettent de prédire la décision de retenir le signalement : la source du signalement, la problématique identifiée, la personne mise en cause et l'unité administrative ayant reçu le signalement. Les trois premiers facteurs, auxquels s'ajoute le statut de l'enfant dans l'établissement, permettent de prédire l'issue de la décision sur la compromission. Il s'agit de caractéristiques qui se rapportent soit au signalant soit à la situation et au traitement administratif des signalements. De manière générale, les caractéristiques de l'enfant signalé et de son milieu de vie (âge, sexe, origine ethnique, type de famille), comme les caractéristiques des ressources impliquées au moment du signalement (intervention d'une ressource psychosociale ou médicale, signalement à la police), ne permettent pas de prédire l'issue des décisions rendues. Dans l'ensemble, il appert que les résultats de nos analyses convergent largement et que les facteurs qui s'avèrent significatifs jouent un rôle important aux étapes de réception et d'évaluation des signale- 
ments. Toutefois, et il est essentiel de le souligner, ces facteurs n'interviennent pas toujours de la même façon aux deux étapes étudiées.

\section{Discussion}

\section{La source du signalement}

Les résultats obtenus dans notre étude font d'abord ressortir l'influence de la source du signalement sur les deux décisions étudiées. À l'instar de plusieurs recherches menées sur le sujet (Groeneveld et Giovannoni, 1977 ; DiLeonardi, 1980 ; Eckenrode et al., 1988a, 1988b ; Flango, 1991 ; Winefield et Bradley ; 1992 ; Trocmé et al., 1994 ; Drake, 1995 ; Wells et al., 1995 ; Zuravin et al., 1995), on observe des différences entre signalants professionnels et non professionnels, différences qui sont cependant plus marquées à l'étape de la réception des signalements.

Par ailleurs, il est important de souligner l'existence de différences entre les diverses catégories de professionnels ainsi qu'entre les nonprofessionnels. À l'étape de la réception des signalements, les intervenants des centres jeunesse et des centres d'accueil se distinguent très nettement de toutes les autres catégories de signalants. Ces intervenants connaissent mieux que quiconque les situations qui requièrent une prise en charge des services de protection et sont à même de faire valoir aux intervenants chargés de la réception des signalements les éléments justifiant qu'une évaluation de la situation de l'enfant soit réalisée. Leurs signalements ont cependant plus de chances de s'avérer non fondés que ceux de la majorité des autres catégories de signalants professionnels, révélant, selon nous, que, pour ces intervenants, c'est plutôt à l'étape de l'évaluation que s'effectue le screening. Les médecins, intervenants en CLSC, en centre hospitalier et en milieu scolaire ont quant à eux plus de chances de voir leurs signalements retenus et jugés fondés. Il semble par conséquent qu'ils signalent, du moins dans une plus grande mesure que les autres déclarants, des situations considérées à plus « haut risque » et pour lesquelles ils disposent déjà d'informations suffisantes pour établir la « matérialité des faits » selon les critères de l'établissement. Il est sans doute plus étonnant de constater que les signalements effectués par les policiers ont une probabilité plus faible d'être retenus que ceux des autres professionnels. Certaines recherches montrent qu'il s'agit d'une catégorie de déclarants dont les taux de signalements fondés sont les plus élevés (Groeneveld et Giovannoni, 1977 ; Eckenrode et al., 1988b ; 
Winefield et Bradley, 1992 ; Trocmé et al., 1994 ; Drake, 1995). On aurait pu s'attendre à des résultats similaires à l'étape de la réception des signalements. Ce qu'il faut sans doute relever, c'est la grande diversité des situations rapportées par les policiers dans notre échantillon (plaintes, appels d'urgence ou d'aide, enfants laissés seuls ou " errants », situations déjà sous enquête policière ou dont l'enquête est terminée...). Il peut notamment s'agir de signalements où des moyens sont déjà mis en œuvre pour corriger la situation ou encore dans lesquels les parents, et le jeune s'il y a lieu, offrent une bonne collaboration. À l'étape de l'évaluation cependant, les signalements des policiers s'avèrent rarement non fondés, ce qui rejoint les résultats des recherches antérieures.

Si on se tourne du côté des signalants non professionnels, les recherches antérieures révèlent généralement que les signalements des voisins et des connaissances (Eckenrode et al., 1988a, 1988b ; Trocmé et al., 1994 ) sont, avec les signalements anonymes (Adams et al., 1982 ; Zuravin et al., 1987 ; Flango, 1991 ; Trocmé et al., 1994 ; Drake, 1995 ; Eckenrode et al., 1998b) et ceux effectués par le parent non gardien (non-custodial parent) (Trocmé et al., 1994 ) ou le parent qui n'est pas mis en cause (non-perpetrating parent) (Hutchison, 1989 ; Wells et al., 1995) ceux qui ont le moins de chances d'être retenus ou jugés fondés. Nos résultats vont dans le même sens à plusieurs égards. Ils accréditent en particulier la thèse selon laquelle les voisins, les connaissances, les membres de la parenté et les pères-signalants rapportent des situations jugées moins sérieuses ou ne possèdent pas toujours l'information suffisante pouvant justifier une évaluation plus en profondeur de la situation. Ajoutons que la crédibilité de ces quatre catégories de signalants est plus fréquemment mise en cause (Jacob,1997), ce qui semble révéler qu'une fraction plus importante de leurs signalements se situent dans le cadre de conflits ou du moins, sont perçus comme tels. À l'étape de l'évaluation, les voisins et les connaissances se distinguent encore une fois de toutes les autres catégories de signalants non professionnels et tout particulièrement des parents. Les pères-signalants ne different pas ici des mères-signalantes. Compte tenu du petit nombre de signalements provenant de pères $(n=16)$, il est toutefois difficile d'évaluer la portée réelle de ce constat. Quoi qu'il en soit, les résultats obtenus dans notre recherche soulignent la pertinence de considérer de façon distincte les diverses catégories de signalants non professionnels et de poursuivre les analyses visant à mettre en lumière leurs particularités. Les différences entre mères-signalants et pères-signalants mériteraient notamment d'être examinées plus en profondeur. 


\section{La nature des problèmes identifiés}

Les décisions prises par les intervenants sont également influencées par la nature des problèmes identifiés. À l'étape de la réception des signalements, ce sont les problèmes d'absentéisme scolaire et les problèmes considérés comme des conflits familiaux qui présentent la probabilité la plus faible d'être retenus. D'une façon générale, la désignation même des problèmes rapportés sous ces problématiques traduit en soi qu'ils ne sont pas jugés comme étant du ressort des services de protection. Giovannoni (1991 : 52) rapporte elle aussi que les signalements qui ne sont pas définis comme des situations d'abus ou de négligence mais qui sont plutôt considérés comme des problèmes familiaux figurent parmi les signalements qui sont habituellement écartés lors du premier screening. L'absentéisme scolaire fait partie des motifs en vertu desquels la sécurité ou le développement de l'enfant peut être jugé compromis (art. 38.1b), toutefois, ce motif est rarement suffisant pour justifier une prise en charge des services de protection. Si l'enfant est jeune, la situation est plutôt définie comme une situation de négligence de la part d'un ou des parents. Si l'enfant est plus âgé, il doit généralement manifester d'autres problèmes de comportement pour que son signalement soit retenu, signalement qui est alors classé sous la problématique des troubles de comportement sérieux. Il s'ensuit que, mis à part quelques exceptions, dans la majorité des cas, les situations d'absentéisme scolaire sont, par définition, jugées moins sérieuses.

À l'autre extrémité, ce sont les signalements pour abus sexuels qui présentent la probabilité la plus élevée d'être retenus. Ce constat contraste avec les résultats obtenus lors de l'étude de la décision sur la compromission où les signalements pour abus sexuels sont ceux qui présentent le plus de chances de s'avérer non fondés, principalement en raison d'une absence d'éléments à l'appui. Ces résultats, qui rejoignent notamment ceux de Hutchison (1989), reflètent à notre avis le statut particulier de ces signalements et le caractère de gravité qui leur est attribué. Comparativement aux autres types de problèmes, le concept d'abus sexuels semble comporter moins d'ambiguiité et faire l'objet d'un consensus relatif. Peu de situations impliquant des activités sexuelles entre adulte et enfant ne sont pas désignées comme des abus. Le fait qu'il s'agisse de gestes isolés ou peu fréquents ou encore d'événements plus lointains ne constituent pas non plus des motifs suffisants pour juger un signalement non recevable. Comme le mentionnent Deisz et al. (1996 : 282) à propos de la perception d'intervenants en protection de l'enfance : «Allegations of sexual abuse, 
no matter how distant or remote in time, were credited special qualities not accorded to other areas of abuse and neglect. » Ces résultats font également ressortir les difficultés particulières liées à l'évaluation des abus sexuels et plus spécifiquement à l'évaluation des abus sexuels intrafamiliaux. D'une part, il peut s'avérer plus difficile d'écarter des doutes d'abus sexuels sans qu'une évaluation plus en profondeur de la situation de l'enfant ne soit réalisée. D'autre part, même à l'étape de l'évaluation, les allégations d'abus sexuels demeurent souvent plus ardues à démontrer, en particulier lorsqu'il s'agit de jeunes enfants. Il est rare que les abuseurs reconnaissent spontanément ce type d'abus ou qu'on puisse disposer de preuves médicales (Waterhouse et Carnie, 1992 ; Bays et Chadwick, 1993 ; Berliner et Conte, 1993 ; Haskett et al., 1995). Si dans certains cas les informations recueillies lors de l'évaluation permettent d'écarter la présomption d'abus, dans d'autres cas toutefois, l'incertitude persiste. Dans notre recherche, les situations d'abus sexuels constituent d'ailleurs, avec les situations de négligence, les signalements pour lesquels les intervenants expriment le plus ouvertement des doutes quant à la décision rendue à l'issue de l'évaluation.

Les problèmes de négligence, de troubles de comportement et d'abus physiques présentent des probabilités similaires d'être retenus. À première vue, il peut sembler un peu surprenant que cette probabilité ne soit pas plus élevée pour les signalements d'abus physiques (Jones, 1996 ; Hutchison, 1989 ; Wells et al., 1991, 1995). Indiquons cependant que dans notre échantillon une part considérable de ces signalements $(40 \%)$ ne mentionnent la présence d'aucune marque ou blessure, conférant une moins grande clarté à ces situations (Hutchison, 1989). Il est en outre important d'indiquer que, dans l'établissement où a été menée l'enquête, les situations «à risque » d'abus physiques (incluant les menaces ou tentative d'homicide) sont généralement classées sous le couvert de la négligence (art. 38), ce qui a sans doute pour effet d'atténuer les différences entre les types d'abus. Contrairement aux résultats de plusieurs recherches (Groeneveld et Giovannoni, 1977 ; Eckenrode et al., 1988a ; Hutchison, 1989 ; Drake, 1995 ; Jones, 1996), les signalements d'abus physiques comportent en outre plus de chances d'être jugés non fondés que les situations de négligence et de troubles de comportement. (Trocmé et al., 1994). Il peut s'agir d'une mauvaise interprétation des faits rapportés ou encore des signalements dans lesquels les méthodes éducatives des parents ne peuvent pas, du point de vue des intervenants, être assimilés à des gestes abusifs. 


\section{La personne mise en cause}

Les résultats obtenus mettent également en évidence l'influence indéniable qu'exerce la personne mise en cause sur la définition donnée à la situation. D'une part, les trajectoires des signalements ne sont pas les mêmes selon que ce soit ou non un membre de la famille qui est mis en cause. La plupart des signalements impliquant un tiers ne sont pas retenus dès l'étape de la réception des signalements et sont orientés vers d'autres instances. Il apparait clairement que la situation de ces enfants n'est prise en charge que si les parents ne sont pas en mesure de protéger l'enfant. Bien que ce constat ne soit pas nouveau, et que plusieurs législations ou politiques en matière de screening excluent explicitement les situations dans lesquelles «the perpetrator is not a caretaker » (Wells et al., 1989 ; Downing et al., 1990), il met en relief que des voies distinctes sont empruntées en réponse aux situations-problèmes signalées selon qu'il s'agisse de tiers ou de membres de la famille.

D'autre part, les trajectoires des signalements ne sont pas les mêmes selon que ce soit le père, la mère, le conjoint d'un parent ou l'enfant luimême qui soit jugé responsable de la situation-problème. Les signalements qui ont le plus de chances d'être retenus et pour lesquels la compromission a le plus de chances d'être établie sont les signalements impliquant la mère et ceux impliquant plus d'une personne (dont la très grande majorité mettent également la mère en cause) ${ }^{8}$. Ces situations peuvent être jugées plus sérieuses, soit parce que plus d'un membre de la famille manifeste des attitudes ou des comportements problématiques, soit parce que, lorsque la mère est la seule en cause, ses attitudes ou comportements apparaissent comme ayant des conséquences plus importantes pour l'enfant. Plusieurs travaux ayant porté sur les mauvais traitements envers les enfants (Breines et Gordon, 1983 ; Martin, 1983 ; Mayer-Renaud et Berthiaume, 1985 ; Gordon, 1988 ; Stark et Flitcraft, 1988 ; Swift, 1991 ; Waterhouse et Carnie, 1992) relèvent que la mère est quasi toujours tenue responsable, soit directement soit indirectement. À notre connaissance toutefois, aucune recherche n'a fait ressortir la

8. Alors que les mères représentent la moitié $(51 \%)$ des personnes mises en cause à l'entrée des services de protection, au terme de l'évaluation, cette proportion s'élève à plus de $73 \%$. En comparaison, les pères sont impliqués dans $27,4 \%$ des situations établissant la compromission de la sécurité ou du développement de l'enfant. Ce pourcentage est de 26,6\% pour les jeunes et de 11,3\% pour les conjoints d'un parent. Relevons également que, dans notre recherche, aucun des signalements impliquant un tiers ou un membre de la parenté n'a franchi l'étape de l'évaluation. 
sélection qui s'opère à l'intérieur même des services de protection selon le sexe du parent mis en cause. Ce constat renvoie bien sûr aux conditions d'exercice des rôles parentaux et met en évidence les positions fondamentalement différentes des hommes et des femmes au sein de la famille. Ces résultats appuient l'hypothèse qu'il existe une gestion ou un traitement différentiel des signalements selon le sexe du parent mis en cause, hypothèse qu'il importerait d'approfondir davantage.

La probabilité que le signalement soit retenu est également plus élevée lorsque les actes reprochés sont imputés à un membre de la parenté ou au conjoint d'un parent. Dans plusieurs cas, il s'agit de situations impliquant implicitement un parent (la mère) considéré comme n'étant pas en mesure de protéger adéquatement l'enfant. À l'étape de l'évaluation toutefois, ces signalements présentent une probabilité plus forte de donner lieu à une fermeture du dossier, en particulier lorsque les parents sont jugés en mesure d'assurer le bien-être de l'enfant et de recourir aux ressources du milieu, si nécessaire.

Les signalements mettant en cause les jeunes comptent parmi ceux qui présentent le moins de chances d'être retenus. Il est permis d'avancer qu'une fraction importante de ces signalements ne sont pas retenus parce que les parents reconnaissent les problèmes, désirent y apporter des correctifs et démontrent, minimalement tout au moins, qu'ils sont en mesure de le faire. Ces familles seront ainsi orientées vers d'autres services sur une base qualifiée de "volontaire ». Si le jeune a plus de 14 ans, son accord est également nécessaire et pourra être déterminant dans la trajectoire qu'il suivra. À l'étape de l'évaluation, des solutions alternatives peuvent sans doute être envisagées dans une proportion moins élevée de signalements.

L'unité administrative ayant reçu le signalement et le statut de l'enfant dans l'établissement

Le fait qu'un signalement soit enregistré par le service de l'urgence sociale plutôt que par le service régulier augmente ses chances d'être retenu, reflétant sans doute le plus grand nombre de situations de crise ou nécessitant une intervention immédiate reçues par cette unité administrative.

Enfin, le fait qu'un enfant soit « connu » au centre jeunesse, c'est-àdire qu'il ait un dossier «actif » ou « classé » dans l'établissement, diminue ses chances de voir son dossier fermé à la suite de l'évaluation. Les situations de « récidive » sont par conséquent jugées comme étant davantage du ressort des services de protection de la jeunesse. 


\section{Limites de la recherche}

Avant de conclure, il importe de faire état des limites de notre recherche. En premier lieu, les analyses statistiques effectuées (analyses de régression logistique) ont porté sur les effets principaux de chacune des variables à l'étude. Comme il a été mentionné précédemment, les interactions entre ces variables n'ont pas été examinées. Il s'ensuit que certaines hypothèses n'ont pas été explorées dans notre recherche. Par exemple, il est permis de penser que l'influence de l'âge differe selon la problématique identifiée (Eckenrode et al., 1998b), hypothèse qui n'a pu être étudiée. Les résultats obtenus concernant l'origine ethnique de l'enfant signalé imposent par ailleurs certaines réserves en raison du nombre peu élevé de signalements impliquant des enfants d'une autre origine ethnique dans notre échantillon. Il en est de même des résultats concernant les catégories de certaines variables qui comportaient des effectifs restreints et pour lesquels, comme il a été mentionné, nous avons dû procéder à des regroupements. Les variables décrivant les caractéristiques des ressources devraient pour leur part être mieux définies sur le plan méthodologique. Ainsi, il serait important de distinguer le type de suivi effectué par les ressources psychosociales et médicales ou encore de pouvoir différencier clairement les situations ayant nécessité une intervention de la police des situations dans lesquelles une plainte a été officiellement portée. Enfin, plusieurs facteurs susceptibles d'influencer les décisions rendues par les intervenants n'ont pas été considérés dans notre recherche. Parmi ces facteurs, mentionnons tout particulièrement le statut socio-économique des familles, qui, en raison des débats qu'il suscite, mériterait une attention particulière. Il en est de même des facteurs liés aux caractéristiques de l'organisation du travail et aux caractéristiques des intervenants. Il serait également intéressant de mener des recherches similaires dans d'autres centres jeunesse et de procéder à des comparaisons entre les établissements.

\section{Conclusion}

En guise de conclusion, formulons quelques commentaires plus généraux qui se dégagent des résultats de le recherche en regard notamment de la proportion relativement restreinte d'enfants pris en charge par les services de protection d'une part et du pourcentage élevé de références d'autre part. Comme le soulèvent Parton et Otway (1995), ces résultats doivent être interprétés à la lumière des transformations majeures qu'ont 
connues les services de protection au cours de la dernière décennie et qui s'inscrivent dans une redéfinition du problème des abus envers les enfants. D'un problème médico-social, la question des abus envers les enfants est devenue un problème socio-légal. Cette redéfinition du problème se traduit à la fois par une modification du rôle des ressources et des professionnels intervenant dans les situations de mauvais traitements et par une sélection des situations perçues comme étant à plus haut risque.

En ce qui a trait au rôle des ressources, les résultats de notre recherche indiquent une double tendance. D'un côté, on se tourne davantage vers les instances judiciaires et ce, à l'intérieur comme à l'extérieur des services de protection de la jeunesse. Il apparait en outre que les pratiques des intervenants sont fortement marquées par la nécessité de recueillir des preuves tangibles à l'appui de leurs décisions, d'où l'importance centrale accordée aux faits. D'un autre côté, on se tourne davantage vers la communauté et les diverses ressources du milieu, comme en témoigne le pourcentage élevé de références.

À partir des résultats obtenus, il est également permis de conclure que les intervenants retiennent effectivement les situations jugées à plus haut risque. Il importe cependant d'ajouter que la notion de risque ne renvoie pas uniquement à la gravité des faits eux-mêmes ou aux conséquences des faits sur l'enfant mais également à l'attitude des personnes impliquées et à l'existence de ressources dans la communauté. Ainsi, des signalements non retenus ou encore des signalements pour lesquels la compromission n'a pas été établie n'impliquent pas nécessairement des situations sans gravité ou de moindre gravité du seul point de vue des faits (Drake, 1996b).

Ces résultats soulignent en particulier la nécessité d'approfondir la connaissance des interrelations et des interfaces entre les services de protection de la jeunesse, le système judiciaire et les différentes ressources d'intervention psychosociales et médicales. Il nous semble de première importance de poursuivre l'étude des facteurs qui façonnent la définition donnée à la situation et qui déterminent les trajectoires des enfants et des familles signalés non seulement à l'intérieur des services de protection mais aussi à travers l'ensemble des ressources et institutions mises en place pour répondre aux problèmes de l'enfance « en danger $»$. 


\section{Références}

Adams, W., Barone, N. et ToOman, P. 1982. "The Dilemma of Anonymous Reporting in Child Protective Services » Child Welfare 61 (1) : 3-14.

Agresti, A. 1990. Categorical Data Analysis, Toronto : John Wiley et Sons.

Alter, C. F. 1985. «Decision-Making Factors in Cases of Child Neglect » Child Welfare 64 : 99-11.

Bays, J. et Chadwick, D. 1993. "Medical Diagnosis of the Sexually Abused Child » Child Abuse and Neglect 17 : 91-110.

BERliner, L. et CONTE, J. R. 1993. «Sexual Abuse Evaluations: Conceptual and Empirical Obstacles » Child Abuse and Neglect 17 : 111-125.

BREINES, W. et GORDON, L. 1983. "The New Scholarship on Family Violence » Signs 8 (3) : 490-531.

Callahan, M. et Lumb, C. 1983. " My Cheque and my Children: The Long Road to Empowerment in Child Welfare » Child Welfare 74 (3) :795-819.

DeISZ, R., DoueCK, H.J. et GeORge, N. 1996. "Reasonable Cause: A Qualitative Study of Mandated Reporting » Child Abuse and Neglect 20 (4) : 275-287.

Deleury, E., LindSAY, J. et RiveST, M. 1985. Grandir à l'ombre de la famille et de l'État. Étude des intervenants de la Direction de la Protection de la Jeunesse, région 03, Québec : Université Laval.

DiLeonard, J.W. 1980. «Decision Making in Protective Services » Child Welfare 59 (6) : 356-364.

Downing, J.D., Wells, S.J. et Fluke, J. 1990. «Gatekeeping in Child Protective Services: A Survey of Screening Policies » Child Welfare 69 (4) : 357-369.

DraKe, B. 1995. "Associations Between Reporter Type and Assesment Outcomes in Child Protective Services Referrals » Children and Youth Services Review 17 (4) : 503-522.

DRAKE, B. 1996a. "Predictors of Preventive Services Provision Among Unsubstantiated Cases » Child Maltreatment 1 (2) : 168-175.

DrAKE, B. 1996b. «Unraveling Unsubstantiated » Child Maltreatment 1 (3) : 261-271.

DRAKE, B. et PANDY, S. 1996. "Understanding the Relationship Between Neighborhood Poverty and Specific Types of Maltreatment »Child Abuse and Neglect 20 (11) : 1003-1018.

Eckenrode, J., Munsch, J., Powers, J. et Doris, J. 1988a. " The Nature and Substantiation of Official Sexual Abuse Reports » Child Abuse and Neglect 12 : 311-319.

Eckenrode, J., Powers, J., Doris, J., Munsch, J. et Bolger, N. 1988b. "Substantiation of Child Abuse and Neglect Reports » Journal of Consulting and Clinical Psychology 56 (1) : 9-15.

FlaNGO, V. E. 1991. "Can Central Registries Improve Sustantiation Rates in Child Abuse and Neglect Cases? » Child Abuse and Neglect 15 (4) : 403-413. 
Freeman, J.B., Levine, M. et DoueCK, H.J. 1996. « Child Age and Caseworker Attention in Child Protective Services Investigations » Child Abuse and Neglect 20 (10) : 907-920.

GelLes, R. J. 1979. «The Social Construction of Child Abuse », Pp.145-157 in Child Abuse and Violence, sous la direction de D. Gil. New York, N.Y. : AMS Press.

GiOVANNONI, J.M. 1989. « Substantiated and Unsubstantiated Reports of Child Maltreatment » Children and Youth Services Review 11 (4) : 299-318.

GiovanNONI, J.M. 1991. "Unsubstantiated Reports: Perspectives of Child Protection Workers » Child and Youth Services 15 (2) : 51-62.

Giovannoni, J.M. et BeCerra, R. M. 1979. Defining Child Abuse, New York : The Free Press.

Gordon, L. 1988. Heroes of their Own Lives. The Politics and History of Family Violence, NewYork : Viking Press.

Groeneveld, L.G. et Giovannoni, J.M. 1977. « Disposition of Child Abuse and Neglect Cases » Social Work Research and Abstracts 13 : 24-30.

Haskett, M.E., Wayland, K., Hutcheson, J.S. et Tavana, T. 1995. « Substantiaton of Sexual Abuse Allegations: Factors Involved in the DecisionMaking Process » Journal of Child Sexual Abuse 42 (2) : 19-47.

HaWkins, W.E. et DunCAN, D.F. 1985. « Perpetrator and Family Characteristics Related to Child Abuse and Neglect: Comparison of Substantiated and Unsubstantiated Reports » Psychological Reports 56 : 407-410.

Hosmer, D.W. et Lemeshow, S. 1989. Applied Logistic Regression, New York : John Wiley et Sons.

HutChison, E.D. 1989. « Child Protective Screening Decisions: An Analysis of Predictive Factors » Social Work Research and Abstracts 25 (3) : 9-15.

JACOB, M. et LABERGE, D. 1997. « Les déterminants de l'intervention auprès des enfants en danger » Déviance et Société 21 (4) : 443-470.

JONES, L. 1993. " Decision Making in Child Welfare: A Critical Review of the Literature » Child and Adolescent Social Work Journal 10 (3) : 241-262.

JONES, R. 1996. «Decision Making in Child Protection » British Journal of Social Work 26 : 509-522.

Kotch, J.B. et ThOMAS, L.P. 1986. « Family and Social Factors Associated with Substantiation of Child Abuse and Neglect Reports » Journal of Family Violence 1 (2) : 167-179.

Manseau, H. 1990. L'abus sexuel et l'institutionnalisation de la protection de la jeunesse, Sillery: Presses de l'Université du Québec.

MARTIN, J. 1983. «Maternal and Paternal Abuse of Children. Theoretical and Research Perspectives », Pp.293-304 in The Dark Side of the Families, sous la direction de D. Finkelhor et al. Beverly Hills : Sage Publications.

MAyer-Renaud, M. et Berthiaume, M. 1985. Les enfants du silence. Revue de la littérature sur la négligence à l'égard des enfants, Montréal : Centre de services sociaux du Montréal métopolitain. 
Menard, S. 1995. Applied Logistic Regression Analysis, Thousand Oaks, CA : Sage Publications.

Parton, N. et Otway, O. 1995. "The Contemporary State of Child Protection Policy and Practice in England and Wales " Children and Youth Services Review 5-6 : 599-617.

PARTON, N. 1979. "The Natural History of Child Abuse: A Study in Social Problem Definition » British Journal of Social Work 9 : 431-451.

Pforl, S. 1977. «The «Discovery» of Child Abuse » Social Problems 24 (3) : 310-323.

RoBin, M. 1991. "The Social Construction of Child Abuse and «False Allegations» » Child and Youth Services 15 (2) : 1-34.

SMITH, S.L., Sullivan, Q.E. et CoHEN A.H. 1995. « Factors Associated with the Indication of Child Abuse Reports » Journal of Social Service Research 21 (1) : 15-34.

Stark, E. et FlitCRAFT, A. H. 1988. « Women and Children at Risk: A Feminist Perspective on Child Abuse » International Journal of Health Services 18 (1) : 97-118.

SWIFT, K. 1991. "Contradictions in Child Welfare: Neglect and Responsibility », Pp.234-271 in Women's Caring. Feminist Perspectives on Social Welfare, sous la direction de C. Baines, P. Evans et S. Neysmith. Toronto : McClelland et Stewart.

THORPE, D. 1994. Evaluating Child Protection, Buckingham : Open University Press.

TOURIGNY, M. et BOUCHARD, C. 1994. «Incidence et caractéristiques des signalements d'enfants maltraités : comparaison interculturelle »Child Abuse and Neglect 18 (10) : 797-818.

Trocmé, N., McPheE, D., KwoK K.T. et Hay, T. 1994. Ontario Incidence Study of Reported Child Abuse and Neglect, Toronto: The Institute for the Prevention of Child Abuse.

Waterhouse, L. et Carnie, J. 1992. «Assessing Child Protection Risk » British Journal of Social Work 22 (1) : 47-60.

Wells, S.J., Downing, J. et FluKe, J. 1991. « Responding to Reports of Child Abuse and Neglect » Child and Youth Services 15 (2) : 63-72.

Wells, S.J., Fluke, J. et Brown, C.H. 1995. «The Decision to Investigate: Child Protection Practice in 12 Local Agencies " Children and Youth Services Review 17 (4) : 523-546.

Wells, S.J., Stein, T.J., Fluke, J. et Downing, J. 1989. « Screening in Child Protective Services » Social Work 34 (1) : 45-48.

Winefield, H.R. et Bradley, P.W. 1992. «Substantiation of Reported Child Abuse or Neglect: Predictors and Implications »Child Abuse and Neglect 16 (5) : 661-672.

WolocK, I. 1982. "Community Characteristics and Staff Judgements in Child Abuse and Neglect Case » Social Work Research and Abstracts 18 (2) : 9-15. 
Zuravin S.J., Orme, J.G. et Hegar, R. 1995. « Disposition of Child Physical Abuse Reports: Review of the Literature and Test of a Predictive Model » Children and Youth Services Review 17 (4) : 547-566.

Zuravin, S.J., Watson, B. et EhrensChaft, M. 1987. « Anonymous Reports of Child Physical Abuse: Are They as Serious as Reports from Other Sources? » Child Abuse and Neglect 11 (4) : 521-529. 